\title{
Adaptive Control Design for Hysteretic Smart Systems
}

\author{
Xiang Fan* and Ralph C. Smith ${ }^{\dagger}$ \\ Center for Research in Scientific Computation \\ Department of Mathematics \\ North Carolina State University \\ Raleigh, NC 27695
}

\begin{abstract}
Ferroelectric and ferromagnetic actuators are being considered for a range of industrial, aerospace, aeronautic and biomedical applications due to their unique transduction capabilities. However, they also exhibit hysteretic and nonlinear behavior that must be accommodated in models and control designs. If uncompensated, these effects can yield reduced system performance and, in the worst case, can produce unpredictable behavior of the control system. One technique for control design is to approximately linearize the actuator dynamics using an adaptive inverse compensator that is also able to accommodate model uncertainties and error introduced by the inverse algorithm.

This paper describes the design of an adaptive inverse control technique based on the homogenized energy model for hysteresis. The resulting inverse filter is incorporated in an $\mathcal{L}_{1}$ control theory to provide a robust control algorithm capable of providing high speed, high accuracy tracking in the presence of actuator hysteresis and nonlinearities. Properties of the control design are illustrated through numerical examples.
\end{abstract}

Keywords: smart materials, hysteresis, inverse compensation, $\mathcal{L}_{1}$ adaptive control

\section{INTRODUCTION}

A growing emphasis in advanced control system design focuses on the use of multi-functional materials, such as piezoceramics, magnetostrictives, and shape memory alloys, in integrated transducers to improve control authority and performance, reduce weight, and minimize power requirements. For example, piezoceramic compounds have both actuator and sensor capabilities due to the direct and converse piezoelectric effects, microscale set point accuracy, and high frequency $(\mathrm{kHz})$ operating capabilities. This has led to their use in applications ranging from nano-positioning mechanisms in an atomic force microscope (AFM) [6] to inertial sensors in an accelerometer. Magnetostrictive materials also offer both actuator and sensor capabilities as well as broadband transduction and large output forces. Recent applications and products exploiting these properties include high accuracy, high speed milling devices [8] and torque sensing for steering systems [7]. Shape memory alloys operate at lower frequencies ( $<100 \mathrm{~Hz}$ for bulk materials) but offer the largest power densities of the three compounds. Hence they are being considered for applications ranging from vibration dissipation in buildings and flexible aerospace structures to active shape configuration of an airfoil to optimize flight characteristics while minimizing $\operatorname{drag}[5,7]$.

One of the major challenges associated with employing these materials is determining how to compensate for the inherent nonlinear and hysteretic constitutive behavior. The fact that the hysteresis and nonlinearities are rate-, stress-, and temperature-dependent complicate the challenge of high performance high accuracy control. Whereas these nonlinear effects can be minimized by restricting input field levels for certain applications, moderate to high input fields are typically necessary in high performance applications. This necessitates the development of robust control designs which incorporate the hysteresis in a manner which achieves specified

\footnotetext{
*E-mail: xfan2@ncsu.edu;

†E-mail: rsmith@eos.ncsu.edu, Telephone: 919-515-7552
} 
tracking, damping and general performance criteria while employing the transducer materials in the nonlinear regimes which provide them with their unique actuator and sensor capabilities.

Two general strategies are typically considered when designing control laws for hysteretic actuators. One is to employ nonlinear constitutive models to construct nonlinear control designs that accommodate the hysteresis and constitutive nonlinearities. A second strategy is to employ the models to construct inverse representations or compensators which can be employed as filters before the hysteretic actuator in the manner depicted in Figure 1. While such compensation is never exact due to discretization and modeling errors, this mismatch between inverse and hysteretic device can be designed to be small thus significantly reducing the disturbance associated with the transducer. It is the latter strategy that we consider here.

The emphasis in this paper focuses on the incorporation of physics through energy-based techniques to provide control designs capable of achieving stringent tracking and speed criteria. Secondly, we emphasize the development of model-based control designs having the capability for real-time implementation at the speeds dictated by eventual applications. We rely on $\mathcal{L}_{1}$ control theory $[3,4]$ to provide a start point from which to initiate adaptive control designs that incorporate the physical mechanisms which produce hysteresis through inverse compensators.

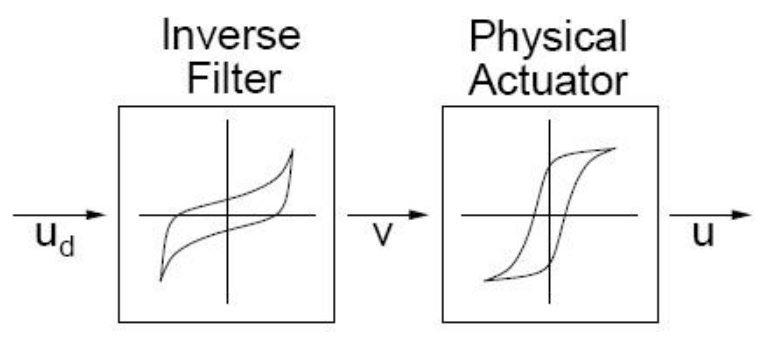

Figure 1. Approximate model inverse employed as a filter for adaptive control design in hysteretic systems.

\section{MODEL DEVELOPMENT}

The key to constructing an inverse compensator that accommodates hysteresis and constitutive nonlinearities is the development of material models which can be efficiently inverted. The material model employed in the present analysis incorporates mesoscopic material behavior at the domain in a stochastic homogenization framework to predict macroscopic material behavior. More specifically, it is constructed in two steps; first, Helmholtz and Gibbs energy relations at the lattice level are used to characterize the local field-polarization/magnetization and field-strain behavior for thermally inactive and active operating regimes; In the second, material nonhomogeneities and variable effective field effects are incorporated through the assumption that ceratin material properties are manifestations of underlying distributions rather than constants.

\subsection{Homogenized Energy Model}

Equations governing the homogenized energy model are summarized here in the context of ferroelectric compounds. Analogous relations hold for ferromagnetic compounds. A detailed review of the modeling framework is given in [7]. The homogenized energy model is based on an energy description at the mesoscopic length scale. This local energy formulation is used to predict macroscopic behavior using a stochastic representation of material nonhomogeneities.

For ferroelectric materials, the Gibbs free energy at the mesoscopic length scale is

$$
G=\psi-E P
$$


where $\psi$ is the Helmholtz energy approximated by the piecewise quadratic function

$$
\psi(P)= \begin{cases}\eta\left(P+P_{R}\right)^{2} / 2, & P \leq-P_{I} \\ \frac{\eta}{2}\left(P_{I}-P_{R}\right)\left(\frac{P^{2}}{P_{I}}-P_{R}\right), & |P|<P_{I} \\ \eta\left(P-P_{R}\right)^{2} / 2, & P \geq P_{I}\end{cases}
$$

Here $E$ is the electric field, $P$ is the polarization, $P_{I}$ denotes the positive inflection point at which the switch occurs, $P_{R}$ is the local remanence polarization and $\eta$ is the reciprocal slope $\frac{\partial E}{\partial P}$. The one-dimensional Helmholtz energy function is double-well potential below the Curie point $T_{c}$ which gives rise to a stable spontaneous polarization with equal magnitude in the positive and negative directions. More details can be found in [7].

The Boltzmann relation gives rise to the local expected values

$$
\begin{aligned}
\left\langle P_{+}\right\rangle & =\frac{\int_{P_{I}}^{\infty} \exp \left(-G\left(E+E_{I}, P\right) V / k T\right) d P}{\int_{P_{I}}^{\infty} P \exp \left(-G\left(E+E_{I}, P\right) V / k T\right) d P} \\
\left\langle P_{-}\right\rangle & =\frac{\int_{-\infty}^{-P_{I}} \exp \left(-G\left(E+E_{I}, P\right) V / k T\right) d P}{\int_{\infty}^{-P_{I}} P \exp \left(-G\left(E+E_{I}, P\right) V / k T\right) d P}
\end{aligned}
$$

of the polarization associated with positive and negatively oriented dipoles, respectively. Here $V$ is the volume of the mesoscopic layer, $k$ is Boltzmann's constant, and $T$ is the temperature.

The local polarization variants are defined by a volume fraction of variants $x_{+}$and $x_{-}$having positive and negative orientations, respectively. The relation $x_{-}+x_{+}=1$ must hold for the volumes fraction of polarization variants.

The resulting local average polarization is qualified by the relation

$$
\bar{P}=x_{+}\left\langle P_{+}\right\rangle+x_{-}\left\langle P_{-}\right\rangle .
$$

The macroscopic polarization is computed by assuming a distribution of local variants thus yielding the relation

$$
[P(E)](t)=\int_{-\infty}^{\infty} \int_{0}^{\infty} \nu_{c}\left(E_{c}\right) \nu_{I}\left(E_{I}\right) \bar{P}\left(E+E_{I} ; E_{c} ; x_{+}\right) d E_{I} d E_{c}
$$

where $\nu\left(E_{c}\right), \nu\left(E_{I}\right)$ respectively denote the distributions of coercive field $E_{c}$ (at which a dipole changes its orientations) and interaction field $E_{I} . x_{+}$represents the distribution of the local variants. The densities can often be modeled as lognormal or normal distributions. However, when more accurate model predictions are critical, a general density can be fit to data. The integrals in (2.6) are solved numerically with quadrature; i.e.,

$$
[P(E)](t)=\sum_{i=1}^{N_{c}} \sum_{j=1}^{N_{I}} \nu_{c}\left(E_{c}^{i}\right) \nu_{I}\left(E_{I}^{j}\right) \bar{P}\left(E+E_{I}^{j} ; E_{c}^{i} ; x_{+}^{i j}\right) w_{c}^{i} w_{I}^{j}
$$

where $w_{c}$ and $w_{I}$ give the quadrature weights. The model for ferromagnetic compounds is equivalent [7].

\subsection{Inverse Compensator}

As discussed in Section 1, one method to accurately control a smart material actuator involves the use of an inverse compensator. The material is nonlinear, hysteretic, and time-varying, but these attributes are approximately linearized by the inverse filter $\widehat{\mathcal{H}}^{-1}$. The composite system to be controlled is then approximately linear and time-invariant. This allows robust control designs, including the $\mathcal{L}_{1}$ control method employed later, to be utilized to accommodate the inversion errors and disturbances. A prototypical setup for this type of control is depicted in Figure 2. For ferroelectric actuators, the control signal $u_{d}$ in Figure 2 is the desired polarization $P$, and input signal $v$ is the electric field $E$ generated by the inverse compensator. 


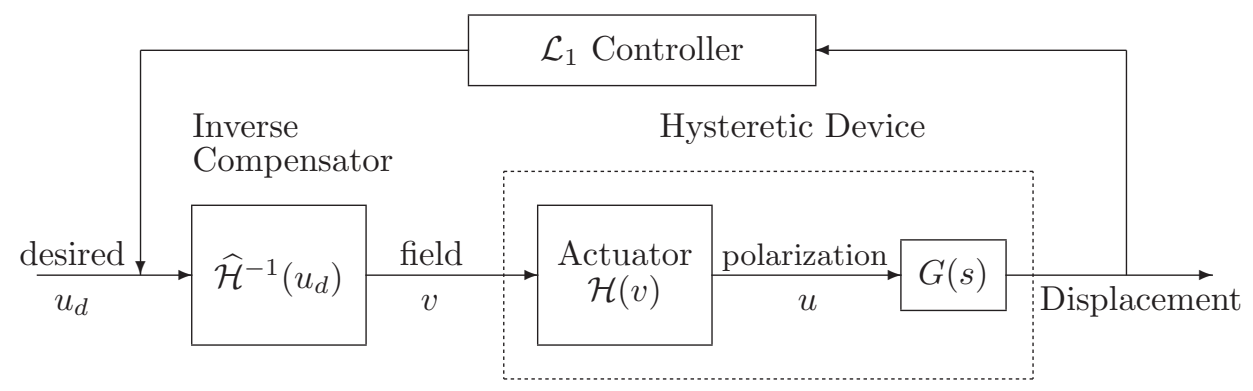

Figure 2. Model-based inverse compensation

The inverse compensator we employ is the inverse of homogenized energy model. To get the inverse representation of (2.6), we need to determine the field level necessary to bring the actuator to the given value of polarization (or strain). More precisely, given any valid state $x_{+}$and any $\widehat{P}$ within the operating range of the material, determine E such that $P=\widehat{P}$, where $P$ is the solution of (2.6). Due to the nonlinearity and dependence on $x_{+}$, it is not feasible to invert (2.6) analytically. Thus, the problem is reformulated as a numerical root finding problem, namely determining the value $E$ such that for a given $x_{+}$and $\widehat{P}$,

$$
P\left(E ; x_{+}\right)-\widehat{P}=0,
$$

Note that if holding internal state $x_{+}$fixed and treating (2.8) as a function solely of $E$, it is a monotone increasing function. However, this function may be discontinuous. More details can be found in $[1,2]$.

\section{MODEL-BASED $\mathcal{L}_{1}$ INVERSE CONTROL ARCHITECTURE}

Consider the system:

$$
\begin{aligned}
\dot{\mathbf{x}}(t) & =\mathbf{A} \mathbf{x}(t)+\mathbf{b} u(t), \quad u(t)=[\mathcal{H}(v)](t) \\
y(t) & =\mathbf{c}^{T} \mathbf{x}(t)
\end{aligned}
$$

where $\mathbf{x}$ is the system state vector. The matrix $\mathbf{A}$ may be unknown or unstable and we assume that there exists a known and stable matrix $\mathbf{A}_{m} \in \mathbb{R}^{n \times n}$ and a vector of ideal parameter $\theta \in \mathbb{R}^{n}$ such that $\left(\mathbf{A}_{m}, \mathbf{b}\right)$ is controllable and $\mathbf{A}-\mathbf{A}_{m}=\mathbf{b} \theta^{T}$. We further assume the (possibly unknown) parameter vector $\theta$ belongs to a given compact convex set $\Theta$. Here $u(t)=[\mathcal{H}(v)](t)$ is the driving force generated by the hysteretic actuator $\mathcal{H}$ where $v(t)$ is the input field to the actuator $\mathcal{H}$.

Next we incorporate the approximate inverse of homogenized energy model as the inverse compensator and use it before the actuator $\mathcal{H}$ (see Figure 2). The driving force $u(t)$ can be rewritten as

$$
u(t)=[\mathcal{H}(v)](t)=\left[\mathcal{H} \widehat{\mathcal{H}}^{-1}\left(u_{d}\right)\right](t)
$$

where the signal $u_{d}(t)$ is used as the input of the inverse compensator $\widehat{\mathcal{H}}^{-1}(\cdot)$ to generate the control field $v(t)$ that is then applied to the hysteretic device.

Substituting (3.2) and the relation $\mathbf{A}-\mathbf{A}_{m}=\mathbf{b} \theta^{T}$ into (3.1) yields

$$
\begin{aligned}
& \dot{\mathbf{x}}(t)=\mathbf{A}_{m} \mathbf{x}(t)+\mathbf{b}\left(u(t)+\theta^{T} \mathbf{x}(t)\right), \quad u(t)=\left[\mathcal{H} \widehat{\mathcal{H}}^{-1}\left(u_{d}\right)\right](t) \\
& y(t)=\mathbf{c}^{T} \mathbf{x}(t) .
\end{aligned}
$$

Our objective is to design a low-frequency adaptive controller $u_{d}(t)$ such that $y(t)$ tracks a given bounded reference signal $r(t)$ while all other error signals remain bounded. 
Note that such model-based inverse compensation is never exact due to the discretization or modeling errors, but the variability due to uncompensated or partially compensated hysteresis and constitutive nonlinearities can be designed to be small thus be assumed bounded in magnitude and be viewed as a structural uncertainty in the actuator model; that is,

$$
u(t)=u_{d}(t)+\sigma(t)
$$

where $\sigma(t)$ is the inversion error and satisfies $|\sigma(t)| \leq \Delta_{0} \in \mathbb{R}$. We will treat the inversion error $\sigma(t)$ as an external time-varying disturbance and attenuate its impact by $\mathcal{L}_{1}$ control techniques [3].

The elements of the $\mathcal{L}_{1}$ adaptive controller are introduced next.

State Predictor: Consider the state predictor

$$
\begin{aligned}
& \dot{\hat{\mathbf{x}}}(t)=\mathbf{A}_{m} \hat{\mathbf{x}}(t)+\mathbf{b}\left(u_{d}(t)+\hat{\theta}^{T} \mathbf{x}(t)+\hat{\sigma}(t)\right), \\
& \hat{y}(t)=\mathbf{c}^{T} \hat{\mathbf{x}}(t) .
\end{aligned}
$$

which has a similar structure as the system in (3.3). The differences are: the unknown parameter vector $\theta(t)$ in (3.3) is replaced by its adaptive estimate $\hat{\theta}(t)$; the driving force $u(t)$ is replaced by the relation (3.4) with inversion error estimate $\hat{\sigma}(t)$. $\hat{\theta}^{T}, \hat{\sigma}(t)$ that are governed the following adaptation laws.

Adaptive Laws: Adaptive estimates are defined via the projection operator

$$
\begin{array}{ll}
\dot{\hat{\theta}}(t)=\Gamma_{c} \operatorname{Proj}\left(\hat{\theta}(t),-\mathbf{x}(t) \tilde{\mathbf{x}}^{T}(t) P b\right), & \hat{\theta}(0)=\theta_{0} \\
\dot{\hat{\sigma}}(t)=\Gamma_{c} \operatorname{Proj}\left(\hat{\sigma}(t),-\tilde{\mathbf{x}}^{T}(t) P b\right), & \hat{\sigma}(0)=\sigma_{0}
\end{array}
$$

where $\tilde{\mathbf{x}}(t)=\hat{\mathbf{x}}(t)-\mathbf{x}(t)$ is the error between the states of the system and the predictor, and $P$ is the solution of the algebraic Lyapunov equation $\mathbf{A}_{m}^{T} P+P \mathbf{A}_{m}=-Q$ where $Q>0$.

Control Law: The control signal $u_{d}(t)$ is generated through gain feedback of the system

$$
\mathcal{X}(s)=D(s) \bar{r}(s), \quad u_{d}(s)=-k \mathcal{X}(s),
$$

where $k \in \mathbb{R}^{+}$is a feedback gain, $\bar{r}(s)$ is the Laplace transformation of

$$
\bar{r}(t)=u_{d}(t)+\hat{\theta}^{T} \mathbf{x}(t)+\hat{\sigma}(t)-k_{g} r(t), \quad k_{g}=-\frac{1}{\mathbf{c}^{T} \mathbf{A}_{m}^{-1} \mathbf{b}}
$$

and $D(s)$ is a transfer function that leads to strictly proper stable

$$
C(s)=\frac{k D(s)}{1+k D(s)}
$$

with low-pass gain $C(0)=1$.

$\mathcal{L}_{1}$-gain stability requirement: Design $D(s)$ and $k$ to satisfy

$$
\|G(s)\|_{\mathcal{L}_{1}} L<1, \quad L=\max _{\theta \in \Theta} \sum_{i=1}^{n}\left|\theta_{i}(t)\right|
$$

where $G(s)=\left(s \mathbb{I}-\mathbf{A}_{m}\right)^{-1} b(1-C(s))$, and $\mathcal{L}_{1}$ gain for a stable proper $m$ input $n$ output transfer function, designated $H(s)$, is defined as

$$
\|H(s)\|_{\mathcal{L}_{1}}=\max _{i=1, \cdots, n}\left(\sum_{j=1}^{m} \int_{0}^{\infty}\left|h_{i j}(t)\right| d t\right)
$$

where $h_{i j}(t)$ is the impulse response of $H_{i j}(s)$, the $i^{\text {th }}$ row $j^{t h}$ column element of $H(s)$. 
In the case of constant $\theta(t)$ and a first order low-pass filter $C(s)=\frac{k}{s+k}$, the stability requirement can be simplified to stabilize the companion system

$$
\begin{aligned}
& \dot{\hat{x}}(t)=\mathbf{A}_{m} \hat{x}(t)+b\left(u_{d}(t)+\hat{\sigma}(t)+\hat{\theta}^{T}(t) x\right) \\
& \dot{u}_{d}(t)=-k u_{d}(t)+k\left(k_{g} r(t)-\hat{\theta}^{T}(t) x(t)-\hat{\sigma}(t)\right)
\end{aligned}
$$

which is reduced to thestability requirement that

$$
A_{g}=\left[\begin{array}{cc}
\mathbf{A}_{m}+\mathbf{b} \theta^{T} & \mathbf{b} \\
-k \theta^{T} & -k
\end{array}\right]
$$

is Hurwitz for all $\theta \in \Theta$.

Note that when $C(s)=1, u_{d}$ reduces to the ideal control signal

$$
u_{i d}(t)=k_{g} r(t)-\theta^{T} \mathbf{x}_{i d}-\sigma(t)
$$

and (3.11) yields the desired system response

$$
\dot{\mathbf{x}}_{i d}(t)=\mathbf{A}_{m} \mathbf{x}_{i d}(t)+\mathbf{b} k_{g} r(t)
$$

by canceling the uncertainties exactly. In the closed-loop predictor system $(3.5)-(3.7), u_{i d}(t)$ is further low-pass filtered by $C(s)$ in (3.9) to have guaranteed low-frequency range. Thus, the system in (3.5) - (3.7) has a different response as compared to (3.12) achieved with (3.11). It has been proved in [3] that the response of the state predictor (3.6) can be made as close as possible to the response of the ideal system (3.12) by making $\|G(s)\|_{\mathcal{L}_{1}}$ arbitrarily small, and $\|G(s)\|_{\mathcal{L}_{1}}$ can be made arbitrarily small by appropriately choosing the design constants, further details may be found in [3] and [4].

\section{SIMULATION}

Consider the dynamic system in (3.1) with $\mathbf{A}=\left[\begin{array}{ll}0 & 1 \\ 5 & 7\end{array}\right], b=\left[\begin{array}{l}0 \\ 1\end{array}\right], c=\left[\begin{array}{l}1 \\ 0\end{array}\right]$ and the state predictor in (3.5) with $\mathbf{A}_{m}=\left[\begin{array}{cc}0 & 1 \\ -10 & -10\end{array}\right]$ and $\theta=\left[\begin{array}{l}15 \\ 17\end{array}\right]$.

We note that $\mathbf{A}$ has poles in the right half plane and hence it is an unstable non-minimum phase system. The constant $\theta$ is assumed to be unknown and the compact set can be conservatively chosen as $\Omega=\left\{\theta_{1} \in\right.$ $\left.[0,25], \theta_{2} \in[0,25]\right\}$. The control objective is to design an adaptive controller $u_{d}(t)$ to ensure that $x_{1}(t)$ tracks any reference signal $r(t)$ both in transient and steady state.

For implementation of the $\mathcal{L}_{1}$ adaptive controller (3.5), (3.6), (3.7), we need to verify the $\mathcal{L}_{1}$ stability requirement in (3.10). Considering the low-pass filter $C(s)=\frac{3 k^{2} s+k^{3}}{(s+k)^{3}}$, we have

$$
G(s)=\left(1-\frac{3 k^{2} s+k^{3}}{(s+k)^{3}}\right) \bar{G}(s),
$$

where $\bar{G}(s)=\left(s \mathbb{I}-\mathbf{A}_{m}\right)^{-1} \mathbf{b}=\left[\begin{array}{ll}\frac{1}{s^{2}+10 s+10} & \frac{s}{s^{2}+10 s+10}\end{array}\right]$.

We choose the conservative $L=50$. It can be verified numerically that $\|G(s)\|_{\mathcal{L}_{1}}=0.0191$ and it follows from (3.10) that $\|G(s)\|_{\mathcal{L}_{1}} L=0.0191 \cdot 50=0.957<1$. Hence, the $\mathcal{L}_{1}$ stability requirement in (3.10) holds.

A typical trajectory common to nano-positioning and industrial applications is plotted in Figure 3. The frequency of the reference signal $r(t)$ is about $70 \pi \mathrm{Hz}$ and it could pass the filter and reference model unchanged. Theoretically, the tracking output $x_{1}(t)$ should exactly match the shape of reference signal after $x_{1}(t)$ converges. 


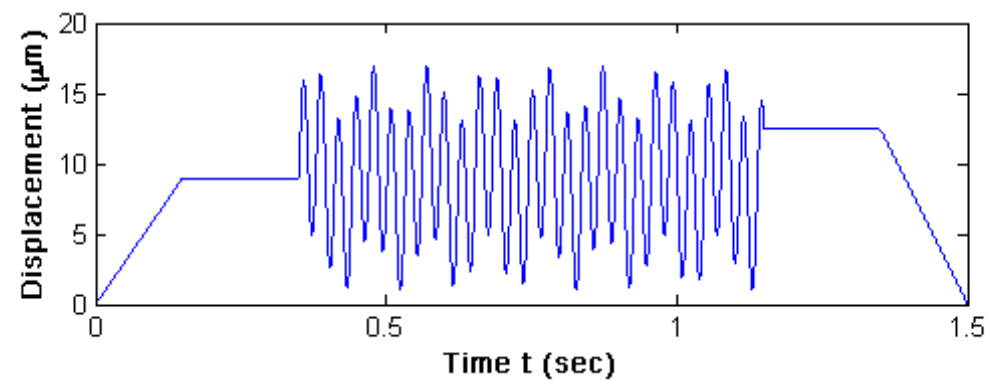

Figure 3. Reference Trajectory $r(t)$

Simulation results are shown in Figure 4. We note that the system output $x_{1}(t)$ converges to $r(t)$ asymptotically whereas $x_{1}(t)$ lags behind the reference signal $r(t)$ with a delay time of about $t_{0}=2 \times 10^{-3}$ sec. The system output $x_{1}(t)$ and predictor output $\hat{x}_{1}(t)$ are almost the same. To cancel the delay, we could redefine $r(t)$ by $r\left(t+t_{0}\right)$, so it requires that we should have an exact prediction of the time-delay $t_{0}$ in advance. The performance with time compensation is shown in Figure 5, and it illustrates that the pre-specified tolerance of $1-2 \mu m$ is achieved in the presence of disturbances and inversion errors.
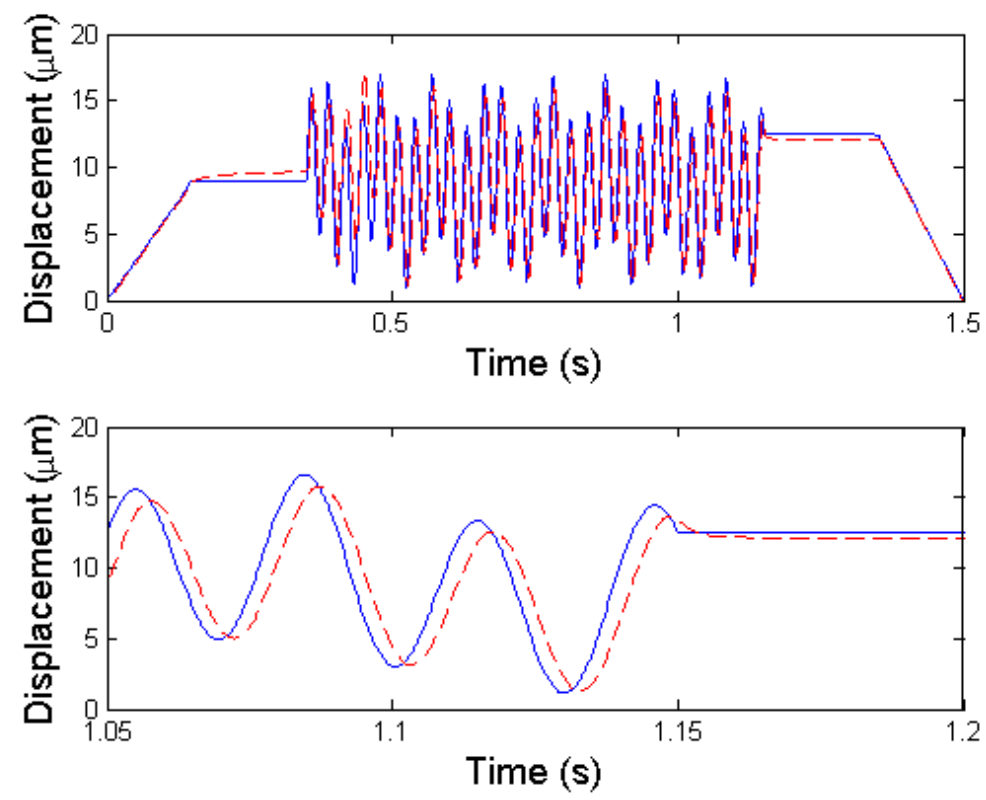

Figure 4. Performance of the $\mathcal{L}_{1}$ adaptive controller for reference input $r=\cos (t)$ without time compensation.

\section{CONCLUSION}

This paper addresses the development of adaptive inverse control designs for high performance smart material transducers operating in nonlinear and hysteretic regimes. When developing models and corresponding inverse compensators, significant emphasis is placed on the utilization of the material's physics to provide the accuracy and efficiency required for real-time implementation of resulting model-based control designs. In the material models, this is achieved by combining energy analysis with stochastic homogenization techniques whereas the 

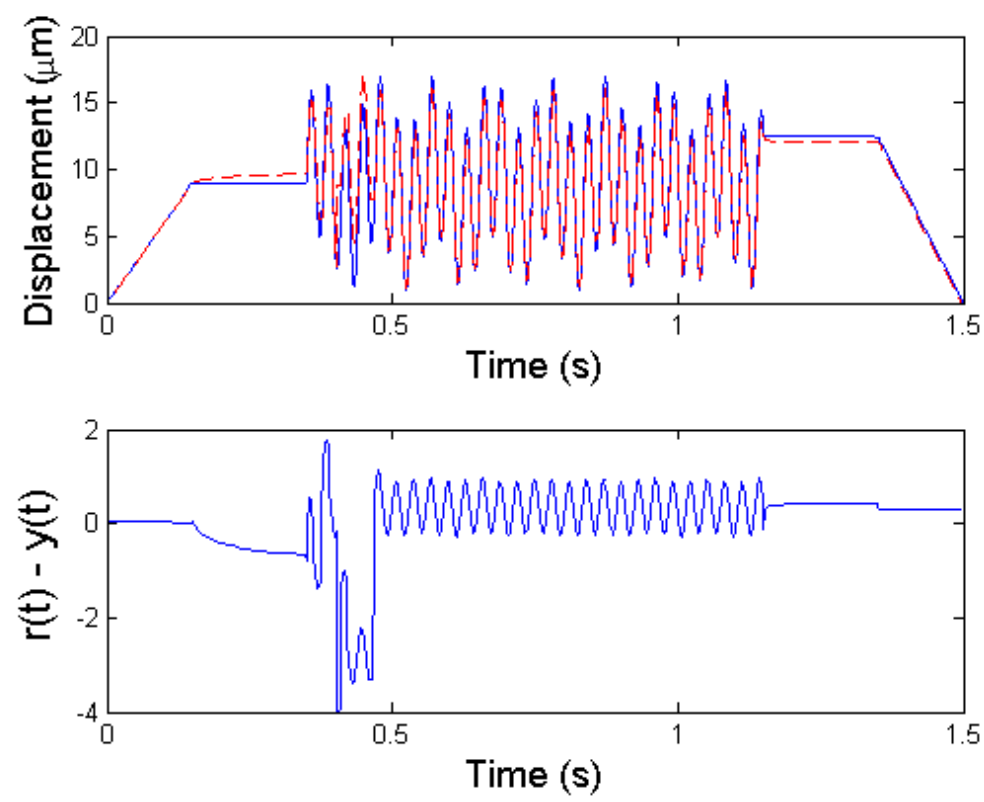

Figure 5. Performance of the $\mathcal{L}_{1}$ adaptive controller for reference input $r=\cos (t)$ with time compensation.

efficiency of forward algorithms is combined with monotonicity properties of the material behavior to provide highly efficient inverse algorithms. These inverse compensators are then incorporated in $\mathcal{L}_{1}$ theory to provide robust control algorithms capable of providing high accuracy tracking even thought the actuators are operating in nonlinear and hysteretic regimes. Through numerical examples, it is illustrated that the $\mathcal{L}_{1}$ robust designs incorporating inverse compensators can achieve the required tracking tolerance.

\section{ACKNOWLEDGEMENT}

This research is supported in part by the Air Force Office of Scientific Research through the grants AFOSRFA9550-04-1-0203 and AFOSR-FA9550-08-1-0348.

\section{REFERENCES}

1. T.R. Braun and R.C. Smith, "High Speed Model Implementation and Inversion Techniques for Ferroelectric and Ferromagnetic Transducers", Journal of Intelligent Material Systems and Structures, 19(11), pp. 1295$1310,2008$.

2. T.R. Braun and R.C. Smith, "Efficient Implementation of Algorithms for Homogenized Energy Models", Continuum Mechanics and Thermodynamics, 18(3-4), pp.137-155, 2006.

3. C. Cao and N. Hovakimyan, "Design and Analysis of a Novel $\mathcal{L}_{1}$ Adaptive Control Architecture with Guaranteed Transient Performance", Proc. American Control Conference, 2006.

4. C. Cao and N. Hovakimyan, " $\mathcal{L}_{1}$ Adaptive Controller for System in the Presence of Unmodelled Actuator Dynamics", Proc. 46th IEEE Conference on Decision and Control, 2007.

5. S. Seelecke and I. Müller, "Shape Memory Alloy Actuators in Smart Structures - Modeling and Simulation", ASME Applied Mechanics Reviews, 56(8), 2003.

6. G. Schitter, P. Menold, H.F. Knapp, F. Allgower and A. Stemmer, "High Performance Feedback for Fast Scanning Atomic Force Microscopes", Review of Scientific Instruments, 72(8), pp. 3320-3327, 2001.

7. R.C. Smith, Smart Material Systems: Model Development. SIAM, Philadelphia, PA, 2005.

8. R.C. Smith, C. Bouton and R. Zrostlik, "Partial and Full Inverse Compenstaion for Hysteresis in Smart Material Systems", Proc. of the American Control Conference, Chicago, IL, pp. 2750-2754, 2000. 